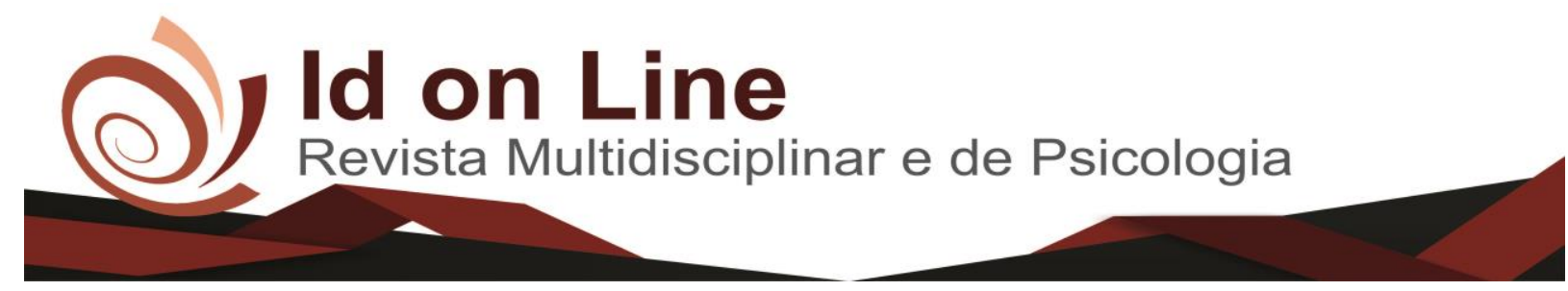

Artigo

\title{
Análise da função sexual e imagem genital em primíparas e multíparas pós-parto vaginal
}

Thamara Brito Silva ${ }^{1}$, Thaynan Rocha Brito de Bulhões ${ }^{2}$, Rosana Porto Cirqueira ${ }^{3}$, Juliana Barros Ferreira ${ }^{4}$

\begin{abstract}
Resumo: o objetivo desse estudo foi analisar e comparar a função sexual e a imagem genital em primíparas e multíparas pós-parto vaginal. Trata-se de um estudo observacional e analítico. Os dados foram resultantes da aplicação de três questionários: um com dados sociodemográficos, o Female Sexual Function Inde (FSFI) que avalia a função sexual, e o Female Genital Self-Image Scale (FGSIS) que avalia a imagem genital. O público acessível foi de 48 mulheres, primíparas e multíparas pós-parto vaginal. A análise dos dados foi realizada através do programa software Statistical Package for the Social Sciences (SPSS) versão 22.0. Foi utilizado o teste do U Mann Whitney para as correlações entre as variáveis do estudo e o teste de qui-quadrado $(\mathrm{p}<0.05)(\mathrm{IC}=0,95)$. O resultado desse estudo mostrou, que as mulheres multíparas apresentaram um percentual menor de disfunção sexual (12\%) do que as primíparas $(34,8 \%)$. No quesito ausência de distorção da imagem genital a amostra das multíparas foi maior que o das primíparas, apresentando 52,0\% e 43,5\% respectivamente, nas primíparas a distorção na imagem genital está correlacionada com a disfunção sexual $(\mathrm{p}<0.05)$. Esse estudo concluiu que mulheres multíparas apresentaram melhores níveis de satisfação sexual e um menor grau de distorção na imagem genital quando comparado às mulheres primíparas. Pouco se sabe da relação que o tipo e número de parto pode influenciar na função sexual, bem como a imagem que a mulher estabelece a sua genitália, sendo necessário mais estudos voltados para essa temática.
\end{abstract}

Palavras-chave: Disfunção sexual fisiológica. Parto natural. Puerpério. Genitália feminina.

\section{Analysis of sexual function and genital image primiparous and multiparous vaginal postpartum}

\begin{abstract}
The aim of this study was to analyze and compare the sexual function and genital image in gilts and multiparous vaginal postpartum. This is an observational and analytical study. The data were the result of the application of three questionnaires: one with socio-demographic data, the Female Sexual Function Inde (FSFI) that evaluates sexual function, and the Female Genital Self-Image Scale (FGSIS) that evaluates the genital image. The accessible public was 48 women, primiparous and multiparous vaginal postpartum. Data analysis was performed using the software Statistical Package for the Social Sciences (SPSS) version 22.0. The Mann Whitney $\mathrm{U}$ test was used for the correlations between the study variables and the chi-square test $(\mathrm{p}<0.05)(\mathrm{CI}=0.95)$. The results of this study showed that multiparous women presented a lower percentage of sexual dysfunction (12\%) than the primiparous women (34.8\%). In the absence of distortion of the genital image, the multiparous sample was larger than that of the primiparous ones, presenting $52.0 \%$ and $43.5 \%$, respectively, in the primiparous genital
\end{abstract}

\footnotetext{
${ }^{1}$ Graduanda em Fisioterapia pela Faculdade Independente do Nordeste-FAINOR, http://www.fainor.com.br, Vitória da Conquista, Bahia, Brasil. E-mail: thamarabrito03@gmail.com

${ }^{2}$ Graduanda em Fisioterapia pela Faculdade Independente do Nordeste-FAINOR, http://www.fainor.com.br, Vitória da Conquista, Bahia, Brasil. E-mail: thaynan.bulhoes@ hotmail.com

${ }^{3}$ Fisioterapeuta, docente da Faculdade de Tecnologia e Ciências-FTC e Faculdade Independente do Nordeste- FAINOR. Mestranda em Saúde Coletiva-UFBA, Brasil. E-mail: porto_rosana@yahoo.com.br

${ }^{4}$ Fisioterapeuta, docente da Faculdade Independente do Nordeste-FAINOR e Faculdade de Tecnologia e Ciências-FTC em Vitória da Conquista/BA. Mestre em Tecnologias em Saúde da EBMSP. E-mail: julibarros78@hotmail.com
} 
image distortion correlated with sexual dysfunction ( $\mathrm{p}<0.05$ ). This study concluded that multiparous women had better levels of sexual satisfaction and a lower degree of distortion in the genital image when compared to primiparous women. Little is known about the relation that the type and number of birth can influence the sexual function, as well as the image that the woman establishes her genitalia, being necessary more studies directed to this subject.

Keywords: Physiological sexual dysfunction. Natural childbirth. Puerperium. Female genitalia.

\section{Introdução}

A sexualidade é caracterizada como um dos principais indicadores de saúde do ser humano, e pode ser compreendida por fatores que exercem uma interação nos aspectos biológicos, psicológicos, culturais, sociais, religiosos e espirituais (VETTORAZZI et al., 2012). Estes aspectos podem influenciar no padrão de vida sexual do ser humano e envolve intimidade, fantasia, erotismo, prazer e reprodução (HOLANDA et al., 2014; MENDONÇA et al., 2012).

Segundo Cavalcanti et al (2014), as queixas na vida sexual podem decorrer durante toda a vida reprodutiva e podem ser afetadas por fatores, tais como, disfunção sexual do parceiro, medicamentos, doenças crônicas, gravidez e paridade. Estas queixas podem interferir no desejo sexual e ocasionar alterações psicofisiológicas e dificuldades interpessoais e contribuir para o surgimento das disfunções sexuais (DS) (PRADO; LIMA; LIMA, 2013).

A disfunção sexual feminina (DSF) é dada como uma desordem no desempenho sexual, envolvendo uma ou mais fases, relacionado com desejo sexual hipoativo, aversão sexual, perturbação da excitação sexual, perturbação do orgasmo, e transtornos dolorosos como dispareunia e vaginismo (MENDONÇA et al., 2012). A prevalência de mulheres com idade de 18 anos ou mais no Brasil apresentando disfunção sexual alcança 49\% (CAVALCANTI et al., 2014; MATHIAS et al., 2015).

O período gestacional, o parto normal e a percepção genital, são fatores que contribuem para o surgimento das DS e torna-se importante compreender as alterações que acontecem com o tipo de parto e a interferência que ocorre na saúde sexual e na concepção da autoimagem genital feminina (AMORIM et al., 2015). A literatura traz a hipótese de que a insatisfação com 
a percepção da imagem genital pode afetar a saúde sexual (BERMAN et al., 2003; ZIELINSKI et al., 2012).

O contentamento da mulher com seu órgão genital externo propicia uma boa função sexual, existindo, dessa forma, fatores que interferem na maneira como as mulheres se sentem diante da satisfação com a sua genitália, sendo influenciadas por questões, sociais, socioculturais e vivencias sexuais (GOMES et al., 2016). Mulheres com autoimagem genital negativa tendem a desenvolver disfunção sexual e uma autoimagem genital positiva favorece na prevenção para algum tipo de transtorno sexual (AMORIM et al., 2015).

Diante do exposto, o propósito do presente estudo buscou analisar a função sexual e imagem genital em primíparas e multíparas pós-parto vaginal; caracterizar o perfil sociodemográfico da população estudada e comparar a função sexual de primíparas e multíparas pós-parto vaginal. Sendo assim, o interesse pelo tema em questão foi contribuir de maneira significativa na vida de mulheres que apresentam alguma disfunção sexual, impossibilitando, portanto, que mantenha uma relação saudável com o seu parceiro e que tenha a autoconfiança com sua genitália após o parto.

\section{Metodologia}

Trata-se de um estudo observacional, com corte transversal e analítico, cuja abordagem é quantitativa.

As mulheres que fizeram parte da pesquisa eram atendidas no Posto de Saúde situada no município de Planalto/Ba. Segundo dados do IBGE (2016), este município possui uma população de 26.743 habitantes, localizado a $471 \mathrm{~km}$ da capital do Estado. A pesquisa ocorreu entre os meses de julho e agosto de 2017.

A amostra foi não probabilística por julgamento. Foram selecionadas mulheres primíparas e multíparas não gestantes, que realizavam acompanhamento ginecológico na unidade no período de coleta da pesquisa. Sendo selecionadas 48 mulheres, dentre elas, 23 primíparas e 25 multíparas.

Os critérios de inclusão deste estudo, foram mulheres com uma faixa etária entre 18 a 45 anos de idade, com vida sexual ativa, e que estivessem devidamente cadastradas no Posto 
de Saúde de Planalto-BA. Quanto aos critérios de exclusão não participaram da pesquisa mulheres que realizaram parto cesáreo, e as que apresentaram dificuldades de compreender e responder o questionário.

Primeiramente foi realizado um contato pessoal com as participantes, onde ficou esclarecido a finalidade e o objetivo da pesquisa, solicitando autorização das referidas mulheres para a coleta dos dados. Após consentimento em participar da pesquisa foi entregue o Termo de Consentimento Livre e Esclarecido (TCLE) e somente após a assinatura do termo, a pesquisa foi iniciada. Após assinar o TCLE, a paciente primeiramente respondeu um questionário com dados sociodemográficos. Em seguida, dois questionários autoaplicáveis, o que avalia a função sexual, o Female Sexual Function Index (FSFI) e o de Imagem Genital, o Female Genital SelfImage Scale (FGSIS). A aplicação dos questionários realizou-se em uma sala disponibilizada pela coordenação do Posto de Saúde.

Os dados sociodemográficos foram coletados em um questionário específico para esse fim, baseado nas orientações do Instituto Brasileiro de Geografia e Estatística - IBGE. Para avaliar a função sexual, foi utilizado o FSFI, este questionário é constituído por 19 questões que avaliam a função sexual nas últimas quatro semanas. Para cada questão existe um padrão de resposta. As opções de respostas recebem pontuação entre 0 a 5 de forma crescente. Invertese a pontuação nas questões sobre dor. Se o escore de algum domínio for igual à zero, significa que não foi referida pela entrevistada relação sexual nas últimas quatro semanas. O resultado da soma dos escores de cada domínio é multiplicada por um fator. $\mathrm{O}$ escore varia de 2 a 36 pontos, se ele for menor ou igual a 26 designa disfunção sexual. O cálculo realizado para os domínios é desejo itens 1 e 2 x (0,6), excitação itens $3,4,5$ e 6 x (0,3), lubrificação itens 7, 8, 9 , e 10 x (0,3), orgasmo itens 11,12 e $13 \times(0,4)$, satisfação itens 14 , 15 e 16 x $(0,4)$, dor itens 17, 18 e 19x (0,4) (PECHORRO et al., 2009).

O questionário FGSIS é composto por 7 itens que avaliam os sentimentos e crenças sobre seu orgão genital. A escala para pontuar é composta de 4 (fortemente, concordo, discordo, totalmente discordo). Cada ítem varia de 7 a 28 pontos. Os escores mais altos indicam uma auto-imagem genital positiva (HERBENICK et al., 2011).

A análise dos dados da pesquisa foi realizada de maneira descritiva, utilizando a tabulação de dados, com média, desvio padrão, porcentagem e frequência através do programa software Statistical Package for the Social Sciences (SPSS) versão 22.0 for Windows. A plotagem das tabelas foi feita por meio do software Microsoft Office Excel 2013 
plus. Foram utilizados o teste do U Mann Whitney para amostras independentes e o teste do qui-quadrado, todos com uma significância de 0,05 (ou confiabilidade de 0,95 ) para observar as correlações entre as variáveis do estudo.

As participantes da pesquisa foram devidamente esclarecidas quanto aos objetivos do trabalho, ficando livres para participar ou não. Uma vez aceitando, assinaram TCLE, sendo respeitados os princípios éticos que constam na resolução 466/12 do Conselho Nacional de Saúde. A desistência ou não participação não implicou em prejuízo ao indivíduo na instituição.

O estudo foi aprovado pelo Comitê de Ética em pesquisa da Faculdade Independente do Nordeste (CEP/FAINOR) conforme protocolo CAAE: 69868717.2.0000.5578 e parecer: 2.132.340.

\section{Resultados}

$\mathrm{Na}$ análise dos dados sociodemográficos verificou-se um grupo de mulheres com idade predominante entre os 29 e 39 anos $(68,8)$, a maioria são casadas $(64,6 \%)$, com ensino médio completo $(47,9 \%)$, desempregadas $(50 \%)$ e vivem com uma renda familiar de até 1 salário mínimo $(54,2 \%)$.Tabela 1

Tabela 1. Características sociodemográficas da amostra. Vitória da Conquista - BA, 2017.

\begin{tabular}{lccc}
\hline Variáveis & \% respostas & $\mathbf{n}$ & \% \\
\hline Quantidade de partos & 100 & & \\
$\quad$ Primíparas & & 23 & 47,9 \\
$\quad$ Multíparas & & 25 & 52,1 \\
Faixa Etária & 100 & & \\
18 - 28 anos & & 13 & 27,1 \\
29 a 39 anos & 33 & 68,8 \\
40 - 50 anos & 2 & 4,2 \\
Raça & 100 & & \\
Branca & & 9 & 18,8 \\
Parda & & 32 & 66,7 \\
Negra & & 6 & 12,5 \\
Indígena & & 1 & 2,1
\end{tabular}


Crença Religiosa

100

Católica

Evangélica

Sem religião

Estado Civil

100

Solteira

2

Casada

Divorciada

31

Viúva

União estável

Mora com companheiro

Não

100

Sim

$\begin{array}{cc}3 & 6,3 \\ 45 & 93,8\end{array}$

Escolaridade

100

Analfabeta

Ensino fundamental incompleto

24,2

Ensino fundamental completo

Ensino médio incompleto

$8 \quad 16,7$

$5 \quad 10,4$

Ensino médio completo

1

2,1

Ensino superior

\section{Situação de trabalho}

100

Desempregada

Aposentada

$\begin{array}{cc}24 & 50,0 \\ 1 & 2,1 \\ 19 & 39,6 \\ 4 & 8,3\end{array}$

Autônoma

\section{Renda Familiar}

Sem rendimento

$3 \quad 6,3$

1 salário mínimo

Até 2 salários mínimos

De 2 a 5 salários mínimos

7

14,6

Fonte: dados da pesquisa.

Os dados encontrados na Tabela 2 referem-se à distribuição da imagem genital e função sexual da amostra. No que se refere à função sexual, 77,1\% das mulheres não apresentam disfunção sexual, já na imagem genital 52,1\% das mulheres apresentam distorção genital. Observa-se que as multíparas possuem um percentual menor em casos de disfunção sexual (12\%), quando comparadas com a quantidade de casos de disfunção em primíparas $(34,8 \%)$. Observa-se também que a maioria das mulheres de ambos os grupos apresentam 
ausência de disfunção sexual, sendo $88 \%$ multíparas e 65,2\% primíparas. Nota-se que as mulheres que tiveram apenas um filho, apresentaram um quadro maior da distorção genital quando comparadas com as que possuem mais de um filho, 56,5\% nas primíparas e $48 \%$ nas multíparas. A ausência de distorção genital na amostra das multíparas foi maior que o das primíparas, apresentando 52,0\% e 43,5\% respectivamente.

Tabela 2. Distribuição da imagem genital e função sexual da amostra. Vitória da Conquista BA, 2017.

\begin{tabular}{|c|c|c|c|c|c|c|c|}
\hline & & \multicolumn{2}{|c|}{$\begin{array}{l}\text { Multíparas } \\
\quad(n=25)\end{array}$} & \multicolumn{2}{|c|}{$\begin{array}{l}\text { Primíparas } \\
(\mathbf{n}=\mathbf{2 3})\end{array}$} & \multicolumn{2}{|c|}{ Total } \\
\hline & & $\mathbf{n}$ & $\%$ & $\mathbf{n}$ & $\%$ & $\mathbf{n}$ & $\%$ \\
\hline \multirow[t]{2}{*}{$F S F I^{1}$} & Disfunção Sexual & 3 & 12 & 8 & 34,8 & 11 & 22,9 \\
\hline & $\begin{array}{l}\text { Ausência de disfunção } \\
\text { sexual }\end{array}$ & 22 & 88 & 15 & 65,2 & 37 & 77,1 \\
\hline \multirow{2}{*}{ FGSIS ${ }^{2}$} & Distorção da imagem genital & 12 & 48,0 & 13 & 56,5 & 25 & 52,1 \\
\hline & $\begin{array}{l}\text { Ausência de distorção da } \\
\text { imagem genital }\end{array}$ & 13 & 52,0 & 10 & 43,5 & 23 & 47,9 \\
\hline
\end{tabular}

${ }^{1}$ Female Sexual Function Index; ${ }^{2}$ Female Genital Self - Image Scale; Fonte: Dados da pesquisa.

Mediante os dados apresentados na Tabela 3, observa-se que as primíparas apenas obtiveram um melhor resultado $(3,99 \pm 1,23)$ em relação às multíparas $(4,34 \pm 1,13)$, no domínio desejo. As multíparas apresentaram melhores médias em todos os demais domínios quando comparadas com as mulheres que tiveram apenas um filho. As médias dos dois grupos (primíparas e multíparas) foram analisadas pelo teste do U Mann Whitney, apontando correlação estatística nos domínios excitação $(p=0,048)$, orgasmo $(p=0,027)$, satisfação $(\mathrm{p}=0,006)$ e na média geral do instrumento $(\mathrm{p}=0,012)$. 
Tabela 3. Função sexual de mulheres primíparas e multíparas. Vitória da Conquista - BA, 2017.

\begin{tabular}{llccc}
\hline & Primíparas & Multíparas & \\
Função Sexual & $\begin{array}{c}\text { Média } \pm \text { Desvio padrão } \\
(\mathrm{n}=23)\end{array}$ & $\begin{array}{c}\text { Média } \pm \text { Desvio padrão } \\
(\mathrm{n}=25)\end{array}$ & \multirow{2}{*}{$p^{*}$} \\
\hline FSFI $^{1}$ Desejo & $3,99 \pm 1,23$ & $4,34 \pm 1,13$ & 0,255 \\
& Excitação & $4,38 \pm 0,78$ & $4,80 \pm 1,0$ & 0,048 \\
Lubrificação & $5,1 \pm 0,97$ & $5,46 \pm 0,87$ & 0,092 \\
Orgasmo & $4,74 \pm 1,08$ & $5,28 \pm 1,16$ & 0,027 \\
Satisfação & $4,73 \pm 1,07$ & $5,42 \pm 0,89$ & 0,006 \\
Dor & $5,06 \pm 1,07$ & $5,56 \pm 0,50$ & 0,33 \\
Média geral & & & \\
do & $28,01 \pm 4,47$ & $30,8 \pm 4,76$ & 0,012 \\
instrumento & & & \\
\hline
\end{tabular}

${ }^{1}$ Female Sexual Function Index; * Teste Mann Whitney; Fonte: Dados da pesquisa.

A imagem genital da amostra apresentada na Tabela 4, dos dois grupos mostra que as primíparas estão melhores no domínio olfato $(3,39 \pm 0,65)$, quando comparadas às multíparas $(3,32 \pm 0,55)$. As médias foram analisadas pelo teste $U$ de Mann Whitney, afim de verificar uma possível correlação estatística entre o grupo de primíparas e multíparas. Desta forma houve correlação estatística entre o domínio orgulho $(\mathrm{p}=0,039)$. Percebe-se que a média geral das primíparas está abaixo de 21,8, demonstrando uma distorção na imagem genital nestas mulheres, o que não ocorre nas multíparas $(22,92 \pm 2,78)$.

Tabela 4. Imagem genital de mulheres primíparas e multíparas. Vitória da Conquista - BA, 2017.

\begin{tabular}{llccc}
\hline & Primíparas & Multíparas & \\
\hline \multirow{2}{*}{ Imagem genital } & $\begin{array}{c}\text { Média } \pm \text { Desvio padrão } \\
(\mathrm{n}=23)\end{array}$ & $\begin{array}{c}\text { Média } \pm \text { Desvio padrão } \\
(\mathrm{n}=25)\end{array}$ & $p^{*}$ \\
\hline Orgulho & $6,04 \pm 1,39$ & $6,84 \pm 0,89$ & 0,039 \\
& Olfato & $3,39 \pm 0,65$ & $3,32 \pm 0,55$ & 0,191 \\
\multirow{2}{*}{ FGSIS } & Aparência & $5,69 \pm 1,52$ & $6,36 \pm 1,22$ & 0,615 \\
& Funcionamento & $5,39 \pm 1,39$ & $6,4 \pm 1,0$ & 0,28 \\
& Média geral do & $20,95 \pm 3,81$ & $22,92 \pm 2,78$ & 0,064 \\
\hline
\end{tabular}

${ }^{1}$ Female Genital Self - Image Scale; * Teste Mann Whitney; Fonte: Dados da pesquisa. 
As informações contidas na Tabela 5, referem-se a um cruzamento de dados da imagem genital por disfunção sexual, sendo utilizado o teste do qui-quadrado de pearson, $(\mathrm{p}<0.05)$. As primíparas apresentaram 13 casos de distorção da imagem genital, 7 com disfunção sexual (53,8\%), e 6 delas tem ausência de disfunção sexual (46,2\%). Verificou-se ainda, nas primíparas, 10 casos de ausência de distorção da imagem genital e destes, $90 \%$ não apresentam disfunção sexual. Com o teste do qui-quadrado de pearson conclui-se que nas primíparas a distorção na imagem genital está correlacionada com a disfunção sexual $(p=0,029)$. Nas multíparas 12 casos indicaram ter distorção na imagem genital, 3 com disfunção sexual (25,0\%), e 9 com ausência de disfunção sexual $(75,0 \%)$. Porém 9 casos na distorção da imagem genital, apresentaram ausência de disfunção sexual (100,0\%). Nas multíparas a distorção da imagem genital não está correlacionada com a disfunção sexual dessas mulheres $(p=0,055)$.

Tabela 5. Referência cruzada imagem genital por disfunção sexual. Vitória da Conquista BA, 2017.

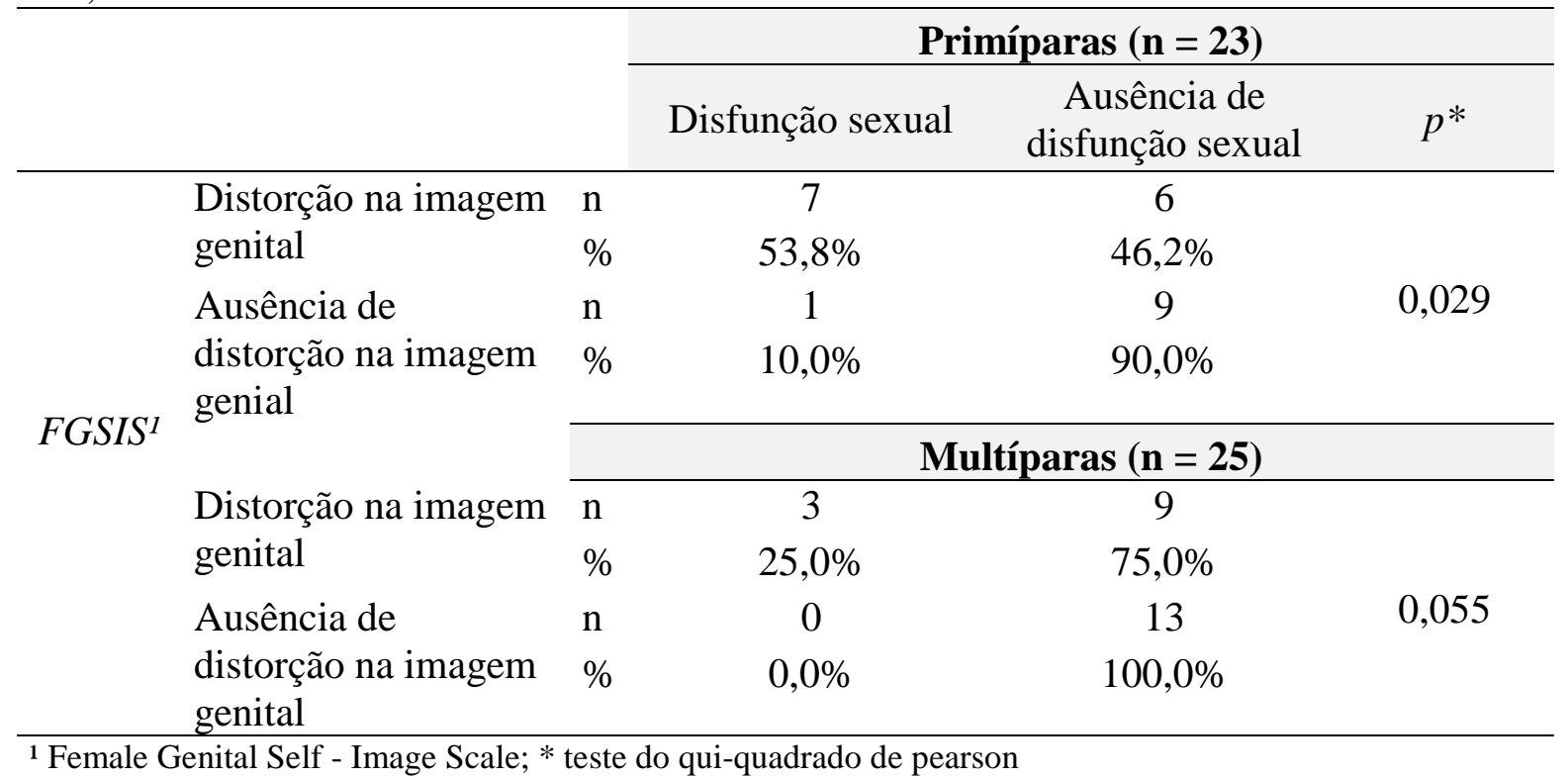

\section{Discussão}

O perfil das mulheres do estudo foi a maioria de adultas, com renda familiar mensal de até um salário mínimo e escolaridade até ensino médio completo. Em relação à renda, os estudos de Mathias et al (2015), mostrou que ao associar a DS com a renda e a escolaridade, não ocorreu 
alterações estatisticamente significativas. Achados do mesmo autor mostraram que o cansaço físico e psicológico vivenciado por mulheres de baixa renda devido as péssimas condições de saúde, trabalho e lazer interferem na qualidade de vida, consequentemente na função sexual.

O estudo de Mendonça et al (2012), mostrou que a prevalência da DSF elevou com o menor nível educacional e a idade, divergindo com os achados da presente pesquisa, onde a maioria das mulheres já concluíram o ensino médio.

Quanto ao estado civil, Cavalcanti et al (2014), aponta que mulheres casadas mostram mais chances de desenvolverem DS, divergindo com a pesquisa em estudo, onde mostrou que 64,6\% dessas mulheres são casadas e que 77,1\% não apresentaram DS. Outro estudo evidenciou que as divorciadas ou separadas apresentaram um índice maior em relação a frequência de disfunção (CABRAL et al., 2013). Sobre a religião, Holanda et al (2014) em seu estudo, mostrou que mulheres católicas e evangélicas apresentaram riscos maiores para a DS do que as sem religião, levando em consideração o fato de serem mulher/mãe, e submissa, dedicando ao seu filho de forma integral, não presenciando de maneira mais efetiva, os prazeres da vida sexual. Fato que não é observado nesta pesquisa.

Os achados do presente estudo evidenciaram que a maioria das primíparas que realizaram parto normal apresentaram alterações na função sexual, e uma percepção negativa da imagem genital. Por essa situação, o estudo de Amorim et al. (2015), concluiu que o número e o tipo de parto têm influência na função sexual feminina, o que não aconteceu com a autoimagem genital.

O fato de optar pelo parto normal espontâneo, não houve diferença significativa na função sexual do estudo em questão. Vetorazzi et al (2012) enfatiza que a relação entre a via de parto e a disfunção sexual, não são exatamente definidas. Além de mostrar que a multiparidade não foi um fator para o desenvolvimento da DS, o que divergem com os achados de Mendonça et al (2012), os quais indicam uma prevalência de DSF que aumenta com a multiparidade, idade e menopausa. E estes fatores são apontados como importantes fatores de risco.

Percebe-se que as primíparas do estudo, apresentaram segundo os dados, menos desejo e um nível maior de dor, que podem estar relacionados com a episiotomia ou não. Mediante tais resultados, Macêdo et al (2017), concluiu em seu estudo que primíparas que realizaram a episiotomia, apresentaram uma pior função no desejo e maiores escores em relação a dor sexual, entretanto as primíparas sem episiotomia demonstraram uma menor função sexual nos domínios excitação, lubrificação, orgasmo e satisfação. Em consonância com esses resultados, 
Silva et al (2012), traz em pesquisa realizada com mulheres brasileiras, o desejo hipoativo e a anorgasmia são a desordem sexual que mais acomete as mulheres.

Em outros estudos, Assis, Rezende e Sá (2015), apontam que disfunções sexuais como dispareunia e redução do desejo sexual estão ligados ao parto normal assistido e não parto normal espontâneo, sendo mais frequentes nos pós-partos mais recentes, enfatizando ainda em outros estudos que independente da escolha do tipo de parto, os níveis de disfunções sexuais no puerpério, apresentaram níveis elevados, e os domínios dor e desejo sexual hipoativo foram os mais significativos. Nos estudos de Holanda et al (2014), destacam ainda que no puerpério os fatores associados para o surgimento de DS, são o tipo de parto, onde o vaginal com episiotomia apresentou riscos maiores, do que a cesariana.

No entanto, como mencionado, o parto cesáreo não é definido como um fator de proteção para a função sexual feminina, e para os músculos do assoalho pélvico (MAP), sendo que não houve diferenças (avaliação de 12 a 18 meses pós-parto) entre mulheres submetidas ao parto vaginal ou cesariana (MENDONÇA et al., 2012). Alguns fatores podem estar associados ao declínio da função sexual no pós-parto, como a amamentação, episiotomia, trauma do períneo devido o parto normal e a laceração vaginal. Durante a amamentação, ocorre aumento da prolactina, redução de andrógenos e estrógenos e liberação da ocitocina. Tais mudanças contribuem para um menor interesse sexual e diminuição da lubrificação vaginal (VETORAZZI et al., 2012).

Sobre essa questão, Assis, Rezende e Sá (2015), enfatizam em um estudo realizado com 315 puérperas, que $61 \%$ das que ainda amamentavam não haviam voltado as suas atividades sexuais no sexto mês após o nascimento do bebê, em comparação a $41 \%$ das que não amamentavam, visto que nos achados de Vettorazzi et al (2012) mostrou em uma amostra de 570 mulheres que o fato de estar no período da amamentação (4 e 12 pós-parto) foi associado a redução da atividade sexual e uma satisfação sexual menor.

$\mathrm{O}$ atual estudo mostrou que a maioria das primíparas apresentou uma maior perturbação orgástica. Este fato pode ter associação com o enfraquecimento dos MAP, o que pode levar a alterações como hipoestesia e anorgasmia. Segundo Mendonça e Amaral (2011), refere que um treinamento desses músculos, auxiliaria em resposta positiva na contração muscular durante o orgasmo, auxiliando na excitação.

De acordo Amorim et al (2015), independentemente do tipo de parto e gravidez, gera modificações para a MAP, devido suportar o peso do feto, visto que, os estudos apontam que o 
parto vaginal sofre mais impacto no MAP, comparados ao cesáreo. O fato de entrar em trabalho de parto, seja qual for a via, pode ocasionar estiramentos nesses músculos (BARBOSA et al., 2015, SILVA 2011). O estudo de Baytur et al (2005), divergem com esses achados, através de um estudo que avaliou a força dos MAP e a função sexual utilizando o FSFI em três grupos de mulheres (pós-parto vaginal, pós-parto cesáreo e nulíparas), não havendo significância sobre a escolha de parto e a função sexual.

Acredita-se que os achados da pesquisa em estudo, podem estar relacionados às modificações pélvicas, devido ter enfrentado a gestação, que podem ocasionar um nível de dor durante a relação sexual, consequentemente, diminuição do desejo. O que pode ser justificado também, em função do corpo das primíparas, que pode não estar adaptado às novas alterações físicas após o nascimento do filho.

Outros importantes dados dessa pesquisa são referentes ao desfecho da percepção da imagem genital em primíparas e multíparas, e se essa percepção perante sua genitália, no parto vaginal, interferiu na função sexual. As primíparas mostraram uma correlação da função sexual com a imagem genital, mas ainda são escassos na literatura estudos que comparem a função sexual com a imagem genital. Berman et al (2003), diz que uma autoimagem genital positiva, as mulheres são vistas como quentes, pessoas apaixonadas, livre para novos relacionamentos e abertas nas questões sexuais.

Mediante tais resultados, um estudo que avaliou a imagem genital através do questionário FGSIS com a quantidade e o tipo de parto em grupos de nulíparas, primíparas e multíparas, mostrou que não ocorreu significância nos escores, apresentando um $p=0,72$. As nulíparas tiveram média de $23,8 \pm 3,4$, as primíparas, $24,1 \pm 3,1$ e as multíparas $23,7 \pm 3,4$. As mulheres que fizeram cesárea obtiveram uma média de $23,8 \pm 3,2$, as que realizaram parto vaginal $24,5 \pm 3,8$ e as que tiveram os dois tipos de parto $23,1 \pm 3,2(p=0,59)$ (AMORIM et al., 2015).

Os estudos mostram que o FGSIS é considerado um questionário confiável para avaliar a imagem genital, no entanto, são necessárias mais pesquisas para a compreensão do questionário em variadas populações (HERBENICK et al., 2010). No entanto, Gomes (2016), traz que o FGSIS apresenta suas limitações, não sendo encontrados outros instrumentos de avaliação para a imagem genital que contemplem todas as necessidades. A falta de um instrumento para avaliar a autoimagem genital em português, dificulta a verificação dos 
preditores para associar a imagem genital, pois parte-se do pressuposto de que o FGSIS pode não representar a verdadeira percepção das mulheres com sua genitália.

Esse estudo apresentou limitações. Uma das limitações foi por ter sido um estudo observacional e não ter realizado a avaliação da força da musculatura pélvica. Outra limitação também foi não ter questionado se foi realizada a episiotomia, ou se estavam amamentando, bem como a inserção de mulheres que realizaram parto cesáreo para comparação dos dados. São necessárias mais pesquisas voltadas para a saúde sexual, para diversificar a população e associar a autoimagem genital e função sexual da mulher após o parto vaginal.

\section{Conclusão}

As mulheres multíparas apresentaram melhores níveis de satisfação sexual e um menor grau de distorção na imagem genital do que as mulheres primíparas, sendo que houve uma correlação na distorção da imagem genital com a disfunção sexual das primíparas. Acredita-se que mais pesquisas com uma amostra maior de mulheres faz-se necessário. Pouco se sabe da relação que o tipo e número de parto pode interferir na função sexual, bem como a imagem que a mulher estabelece a sua genitália, sendo necessário mais estudos que intensifiquem essa temática, com o propósito de diversificar o campo da pesquisa no intuito de verificar esse tipo de relação, principalmente no que se refere a imagem genital.

\section{Referências}

AMORIM, H. et al. Relação do tipo e número de parto na função sexual e autoimagem genital feminina: um estudo observacional. Revista Pesquisa em Fisioterapia. v. 5, n. 1, p. 49-56, abr. 2015.

ASSIS, T.R.; REZENDE, F.R.; SÁ, A.C.A.M. Percepção de puérperas sobre mudanças sexuais após exercícios para o assoalho pélvico: uma abordagem qualitativa. Rev Fisioter $\mathbf{S}$ Fun. Fortaleza, v. 4, n. 1, p. 6-13, jan-jun. 2015.

BELDONE, M.V. Resposta sexual, disfunção sexual e qualidade de vida em mulheres obesas. 2013. 93f. Dissertação. (Mestrado). Faculdade de medicina da universidade de São Paulo, São Paulo, 2013. 
BENUTE, G.R.G. et al. Preferência pela via de parto: uma comparação entre gestantes nulíparas e primíparas. Rev Bras Ginecol Obstet. V. 35, n. 6, p. 281-5, set. 2013.

Berman L.A. et al. Genital self-image as a component of sexual health: Relationship between genital self-image, female sexual function, and quality of life measures. Journal of Sex and Marital Therapy. v. 1, n. 1, p. 11-21, fev. 2003.

CAVALCANTI, I.F. et al. Função sexual e fatores associados à disfunção sexual em mulheres no climatério. Rev Bras Ginecol Obstet. Recife (PE), v. 36, n. 11, p. 497-502, set. 2014.

DELGADO, A.M.; FERREIRA, I.S.V.; SOUSA, M.A. Recursos fisioterapêuticos utilizados no tratamento das disfunções sexuais femininas. Revista científica da escola da saúde. v. 4, n. 1, p. 47-56, jan. 2015.

GOMES, T. et al. IMAGEM CORPORAL E IMAGEM GENITAL FEMININA. Revista científica da escola da saúde. v. 4, n. 2, p.37-42, fev-mai. 2015.

GOMES, T.B.S. Associação entre imagem corporal e imagem genital de mulheres matriculadas em academias: um estudo observacional. 2016. 53 f. Dissertação (Mestrado) Escola Bahiana de Medicina e Saúde Pública Programa de Pós-graduação em Tecnologias em Saúde, Salvador-Ba, 2016.

GIGLIO, M.R.P.; FRANÇA, E.; LAMOUNIER, J.A. Avaliação da qualidade da assistência ao parto normal. Rev Bras Ginecol Obstet. v. 33, n. 10, p. 297-304. 2011.

HERBENICK, D. et al. The Female Genital Self-Image Scale (FGSIS): Results from a Nationally Representative Probability Sample of Women in the United Statesjsm. International Society for Sexual Medicine. v. 8, p. 158-166. 2011.

HOLANDA, J.B.L et al. Disfunção sexual e fatores associados relatados no período pós-parto. Acta Paul Enferm. V. 27, n. 6, p. 573-8, ago. 2014.

LAAN, E. et al. Young women's genital self-image and effects of exposure to pictures of natural vulvas. Journal of psychosomatic obstetrics \& gynecology. p. 1-7, 22 september. 2016.

MACÊDO, L.G. et al. Avaliação da função sexual em primíparas após parto vaginal e nulíparas. Revista Pesquisa em Fisioterapia. v. 7, n. 1, p. 24-29, fev. 2017.

MATHIAS, A.E.R.A. et al. Disfunção sexual: Avaliação de mulheres durante o terceiro trimestre gestacional. ABCS Health Sci. v. 40, n. 2, p. 75-79, abr. 2015.

RIBEIRO, R.; MAGALHAES, A.T.; MOTA, I. Disfunção sexual feminina em idade reprodutiva - prevalência e fatores associados. Rev Port Med Geral Fam. v. 29, p. 16-24. 2013. 
MELO, M.J. A prevalência da disfunção sexual em mulheres adultas atendidas no serviço de ginecologia do hospital universitário Antônio Pedro. 2016. 52f. Dissertação (Mestrado). Universidade Federal Fluminense Escola de Enfermagem Aurora de Afonso Costa curso de graduação em enfermagem e licenciatura, Niteroi, 2016.

MENDONÇA, C.R. et al. Função sexual feminina: aspectos normais e patológicos, prevalência no Brasil, diagnóstico e tratamento. Feminina, v. 40, n.4, p. 196-202, jul/ago. 2012.

MENDOÇA, C.R.; AMARAL, W.N. Tratamento fisioterapêutico das disfunções sexuais femininas: Revisão de Literatura. FEMINA. v. 39, n. 3, p. 139-142, mar. 2011.

MESQUITA, R.L.; CARBONE, E.S.M. Tratamento Fisioterapêutico nas Disfunções Sexuais em Mulheres após Tratamento de Câncer Ginecológico e de Câncer de Mama: Uma Revisão de Literatura. Rev Fisioter S Fun. Fortaleza, v. 4, n. 2, p. 32-4, jul-dez. 2015.

PECHORRO, P. et al. Validação portuguesa do índice de Funcionamento Sexual Feminino (FSFI). Laboratório de Psicologia. v. 7, n. 1, p. 33-44. 2009.

PIRES, D. et al. A influência da assistência profissional em saúde na escolha do tipo de parto: um olhar sócio antropológico na saúde suplementar brasileira. Rev. Bras. Saúde Matern. Infant. Recife, v.10, n. 2, p. 191-197, abr-jun. 2010.

PRADO, D.S.; LIMA, R.V.; LIMA, L.M.M.R. Impacto da gestação na função sexual feminina. Rev Bras Ginecol Obstet, v. 35, n. 5, p. 205-9, mai. 2013.

SILVA, B.M. et al. Incidência de disfunção sexual em pacientes com obesidade e sobrepeso. Rev. Col. Bras. Cir, Alagoas/Maceió, v. 40, n. 3, p.196-202, ago. 2012.

VELHO, M.B. et al. Vivência do parto normal ou cesáreo: revisão integrativa sobre a percepção de mulheres. Texto Contexto Enferm. Florianópolis, v. 21, n. 2, p. 458-66, abr-jun. 2012.

VETTORAZZI, J. et al. Sexualidade e puerpério: uma revisão da literatura. Rev HCPA. v. 32, n. 4, p. 473-479, 27 de nov. 2012.

\section{Como citar este artigo (Formato ABNT):}

SILVA, Thamara B.; BULHÕES, Thaynan R. de; CIRQUEIRA, Rosana P.; FERREIRA, Juliana B. Análise da função sexual e imagem genital em primíparas e multíparas pós-parto vaginal. Id on Line Revista ultidisciplinar e de Psicologia, 2017, vol.12, n.39, p.97-111. ISSN: 1981-1179.

Recebido: 15.11 .2017

Aceito: 18.11 .2017 